\title{
279 ILT3 BLOCKADE REDUCES TUMOR BLAST CELLS IN ACUTE MYELOID LEUKEMIA PBMC AND INHIBITS TUMOR CELL GROWTH IN A HUMANIZED AML TUMOR MODEL
}

${ }^{1}$ Alan Byford*, 'Yujie Qu, ${ }^{1}$ Haiyan Xu, '1Gain Robinson, 'Latika Singh, 'Eric Muise, ${ }^{1}$ Elaine Pinheiro, ${ }^{2}$ Mei Chen, 'Lily Moy, ${ }^{1}$ Stephen Alves, ${ }^{1} J i e$ Zhang-Hoover. ${ }^{1}$ Merck, Boston, MA, USA; ${ }^{2}$ Merck, Kenilworth, NJ, USA

Background Immunoglobulin-like transcript 3 (ILT3), an immune-inhibitory receptor expressed on myeloid cells, is highly expressed in acute myeloid leukemia (AML) with monocytic differentiation (M4 and M5 subtypes) and clinically is negatively correlated with overall survival. We examined the effects of a highly selective and potent chimeric anti-ILT3 $\mathrm{mAb}$ (c52B8) on tumor cell survival/growth and human immune cell modulation in AML PBMC and AML tumor cell lines in pre-clinical in vitro and in vivo models.

Methods ILT3 mRNA and cell surface expression levels were measured by RNAseq and FACS, respectively. PBMC from AML patients with different levels of ILT3 cell surface expression were treated with $\mathrm{c5} 2 \mathrm{~B} 8$ in vitro.A systemic AML model was generated by IV inoculation of NSG mice with luciferasetransfected MV-4-11 human AML cells, with or without human PBMC engraftment. Tumor bearing mice were treated weekly with $\mathrm{c} 52 \mathrm{~B} 8$, modified to contain the human $\mathrm{Fc}$ of either the IgG1 or IgG4 subclass. MV-4-11 growth in vivo was measured by bioluminescence imaging (BLI).CyTOF was performed to phenotype AML PBMC in in vitro culture and bone marrow samples from the systemic MV-4-11 model after the treatment.THP-1 cells/human $\mathrm{T}$ cell co-cultures stimulated with anti-CD3/CD28 antibodies were treated with c52B8. Human $\mathrm{T}$ cell activity was assessed by levels of IFN $\gamma$ production in the culture using MSD ELISA.

Results In ILT3 high AML patient PBMC, c52B8 treatment in vitro decreased the frequency of tumor blasts, modulated Tregs, and increased monocytic myeloid cells. In the MV-4-11 model, weekly treatment with c52B8 significantly reduced tumor burden in vivo, regardless of the $\operatorname{IgG}$ subclass. Importantly, tumor cell growth inhibition by c52B8 occurred in the absence of human PBMC. Additional post-mortem CyTOF analysis of bone marrow cells from MV-4-11/human PBMC inoculated mice confirmed a reduction in MV-4-11 cells, identified by CD3-CD19- human myeloid cells following c52B8 treatment. In THP-1/human $\mathrm{T}$ cell co-cultures, c52B8 treatment reversed THP-1 induced T cell suppression, as measured by enhanced IFN $\gamma$ production.

Conclusions Anti-ILT3 mAb (c52B8) is efficacious in blocking progression of AML with monocytic differentiation by directly influencing tumor cell growth and enhancing human $\mathrm{T}$ cell activity in pre-clinical models. These studies support a potential role for this compound in humans.

Ethics Approval All animal work was reviewed and approved by Merck IACUC before experiments were conducted.

http://dx.doi.org/10.1136/jitc-2021-SITC2021.279 